\title{
Experiences with surgical management of undescended testis at a tertiary care hospital in north-western Tanzania
}

\author{
STEVEN KADILO ${ }^{1}$, MANGE MANYAMA², NEEMA M. KAYANGE ${ }^{3}$ and PHILLIPO L. CHALYA ${ }^{1^{*}}$ \\ ${ }^{1}$ Department of Surgery, Catholic University of Health and Allied Sciences-Bugando, P.O. Box 1464, Mwanza, \\ Tanzania \\ ${ }^{2}$ Department of Anatomy, Catholic University of Health and Allied Sciences-Bugando, Mwanza, Tanzania \\ ${ }^{3}$ Department of Paediatrics, Catholic University of Health and Allied Sciences-Bugando, Mwanza, Tanzania
}

\begin{abstract}
Background: Undescended testes (UDT) are prone to a lot of complications but early detection and correction give good results. There is paucity of published data on UDT in our setting. This study describes the clinical presentation, management and outcome of this condition at Bugando Medical Centre (BMC) in northwestern Tanzania

Methods: This was a retrospective study of patients who were admitted and operated for undescended testis at BMC between July 2006 and June 2014.

Results: A total of 84 patients with 102 UDT were studied. Forty-six (54.9\%) patients were aged above 5 years. Majority (72.5\%) UDT were non-palpable. The right side was involved in $54.8 \%$; left side in $23.8 \%$ while $21.4 \%$ were bilateral. Associated inguinal hernia was reported in 48 (77.4\%) patients. Ultrasonography was performed in 34 (45.9\%) patients with non-palpable testes and was used to locate 12(35.3\%) testes. Laparoscopy was not used in any patient. At surgery, 54 (52.9\%) testes were found in the inguinal canal, 28 (27.5\%) in the superficial inguinal pouch, 10 (9.8\%) in the abdomen and 10 (9.8\%) testes were not found. Out of the 92 (90.2\%) visible testes, 69 (75.0\%) underwent orchidopexy (single stage in 54 (78.3\%) testes and multistage in 15 (21.7\%) testes) and $23(25.0 \%)$ orchidectomy. Single stage procedures were more likely in patients less than 5 years, with palpable testes and those with testes located at the superficial inguinal pouch $(p<0.001)$. Postoperative complications were recorded in $8(9.5 \%)$ patients. The overall success rate of treatment was $95.7 \%$. In this study, only $12(14.3 \%)$ patients were available for follow up at 12 months after discharge.

Conclusion: Patients with UDT presented late in our hospital because diagnosis by birth attendants was rarely made even though the anomaly was obvious at birth. The parents, patients themselves and development of complications drew attention to undescended testis. Health awareness campaign, thorough genital examination after birth and regular screening of toddlers for UDT may result in early presentation

Keywords: Undescended testis, clinical presentation, management outcome, Tanzania
\end{abstract}

\section{Introduction}

Undescended testis (UDT) is a congenital abnormality of the testis whereby its descent into the scrotum is arrested along its normal path (Hutson, 1998; Umakanth \& Menon, 2000; Sekabira et al., 2003). It is a common congenital anomaly worldwide and its incidence tends to fall with increasing age. At birth, the incidence of UDT is around 4\% to 5\% (Hutson, 1998; Ameh \& Mbibu., 2000). This figure falls to $1 \%$ to $2 \%$ at 12 months of age and remains fairly constant at $0.8 \%$ into adulthood (Berkowitz et al., 2003; Seth et al., 2006; Dina et al., 2008). Approximately 20\% of UDTs are impalpable and $10 \%$ are in an intra-abdominal position (Hutson \& Clarke, 2007). UDTs are unilateral in about $90 \%$ of individuals and bilateral in about $10 \%$ (Hutson \& Clarke, 2007).

\footnotetext{
* Correspondence: Phillipo L. Chalya; E-mail: drphillipoleo@yahoo.com
} 
Testes that remain undescended are associated with increasing the incidence of infertility, malignancy, torsion and trauma (David \& lyekoretin, 2008). UDT is associated with progressive loss of germ and Leydig cells. There is a $2 \%$ risk of severe germ cell loss and $1 \%$ risk of Leydig cell depletion for each month a testis remains undescended (Hutson \& Clarke, 2007). The relative risk of testicular cancer in an undescended testis is between 2.75 to 8 that of a normal male. The risk appears higher in patients with bilateral UDT and late (after age of 12) or uncorrected UDT (David \& lyekoretin, 2008). Previous studies have estimated that an UDT increases the risk of testicular torsion by 10 times (Dorit et al., 2006). The mechanism of torsion in UDT is not well understood and the diagnosis can be challenging. Timely treatment before the age of 2 years is advocated to forestall these complications (David \& lyekoretin, 2008).

The diagnosis of palpable UDT is mainly clinical. However, the location of the impalpable testes frequently poses a diagnostic challenge. Ultrasonography gives false negative rates as high as $50 \%$ and is believed not to be any better than careful examination by an experienced surgeon (Cortes et al., 1995). Laparoscopy is currently the most reliable diagnostic modality in the management of impalpable testes. It clearly shows the anatomy and provides visual information upon which a definitive decision can be based (Cortes et al., 1995). However, this form of diagnostic facility is not always available in most centers in resource-limited countries.

The fundamental principle for the management of UDT is operative. The initial treatment of neonates with UDT is typically observation, since it has been estimated that more than $70 \%$ of spontaneous testicular descent occurs in the first 3 month of life (David et al., 2004). Early surgical treatment has a beneficial effect on germ cells function and subsequent 'catch-up' testicular growth (Claude et al., 2007). In developed countries where the level of awareness is high and patients present early, the rate of complications are low. In developing country, however, patients present late to health facilities either following accidental discovery by health workers, parents/caregivers or after complications have taken place (Taha et al., 1990; Ameh \& Mbibu, 2000). Treatment of undescended testis in our setting, therefore, pose a great challenge as the majority of patient present late when the testicular vessels are already shortened, the testis has reduced in volume, became atrophic or may have undergone malignant change or other irreversible complications (Osegbe \& Amaku, 1985). The non availability of facilities to do laparoscopic or microsurgical orchidopexy in most centers in the developing countries further compound the problems (Lindgren et al., 1999; Abolyosr, 2006; El-Anany, 2007).

Despite the association of UDT with increased risk of infertility and testicular cancer in later life, it has not received sufficient attention in our environment. Little has been published on this condition in Tanzania and Bugando Medical Centre in particular. This study was undertaken to describe our own experiences in the surgical management of UDT in our local environment, outlining the presentation, management and outcome of undescended testes at BMC in a northwestern Tanzania and make recommendations that may lead to improved results.

\section{Patients and Methods}

\section{Study design and setting}

This was a retrospective study of patients who were admitted and operated for undescended testis at Bugando Medical Centre (BMC) between July 2006 and June 2014. BMC is located in Mwanza city along the shore of Lake Victoria in the northwestern part of Tanzania. It is a tertiary care and teaching hospital for the Catholic University of Health and Allied Sciences-Bugando (CUHASBugando) and has 1000 beds. BMC is one of the four largest referral hospitals in the country and 
serves as a referral centre for tertiary specialist care for a catchment population of approximately 13 million people from neighboring regions in northwestern Tanzania.

\section{Study population}

The study included all patients aged one year and above who were admitted and operated for undescended testis at Bugando Medical Centre during the period of study. Patients with incomplete data and those whose case files could not be retrieved for analyses were excluded from the study. Data were retrieved from the case files stored in the medical record department and collected using a preformed questionnaire. Data administered in the questionnaire included age of the patient, associated congenital anomalies, scrotal findings, clinical finding of the testis (palpable or not palpable), anatomical side affected, diagnostic facilities, operative findings, operative procedure performed, successive rate and postoperative complications. Successful orchidopexy was defined as a scrotal position of the testis and absence of testicular atrophy at the six month follow-up visit (Stephen, 1995).

\section{Data analysis}

The statistical analysis was performed using the Statistical Package for Social Sciences (SPSS) version 17.0 for Windows (SPSS, Chicago, Illinois, United States). The median (and IQR) and ranges were calculated for continuous variables, whereas proportions and frequency tables were used to summarize categorical variables. Categorical data were analyzed using Chi-square $\left(\chi^{2}\right)$ test, where necessary $\mathrm{p}$-values less than 0.05 were regarded as significant. Multivariate logistic regression analysis was used to determine predictor variables that predicted the outcome.

\section{Ethical considerations}

Ethical approval to conduct the study was obtained from the CUHAS-Bugando/BMC joint institutional ethic review committee before the commencement of the study.

\section{Results}

During the study period, a total of 97 patients underwent surgical treatment for UDT at BMC and these were eligible for the study. Of these, 13 patients were excluded from the study due to incomplete data, leading to a final study population of 84 patients with 102 UDT. The age of patients at presentation ranged from 1 to 34 years with a median of 6 years (+ IQR of 4 to 10 years). The modal age group was $1-5$ years accounting for $45.2 \%$ of patients (Figure 1 ). The majority of patients, $46(54.9 \%)$ were aged above 5 years of age.

Associated congenital malformations were reported in $62(73.8 \%)$ patients, of which congenital inguinal hernia was the most frequent congenital malformation reported in 48 (77.4\%) patients. This was followed by hypospadias in $8(12.9 \%)$ patients and umbilical hernia, Down syndrome and cerebral palsy in $2(3.2 \%)$ patients each respectively. Undescended testes were discovered in $56(66.7 \%)$ patients by the parents, 18 (21.4\%) by patients (self-discovered), 8 (9.5) by birth attendants and $2(2.4 \%)$ due to torsion. There was obvious delay in presentation as the median age of the patients whose undescended testes were discovered by their parents was 4years; selfdiscovered 14 years and due to torsion 16 years. The median age of the patients whose undescended testes were discovered by birth attendants was 2 years. The presenting symptom was empty scrotum in $52(61.9 \%)$, inguinal hernia in 29 (34.5\%) patients, inguinal mass in $2(2.4 \%)$ patients and testicular torsion in $1(1.2 \%)$ patient. Out of 102 UDT, 74(72.5\%) testes were non-palpable and only $28(27.5 \%)$ testes were palpable. Forty-six (54.8\%) patients had right sided UDT and $20(23.8 \%)$ had left 
sided UDT with a right-to-left ratio of 2.3: 1. Eighteen (21.4\%) patients had bilateral UDT. Evaluation of patients was mainly clinical. Ultrasonography was performed in 34 (45.9\%) patients with nonpalpable testes and was used to locate $12(35.3 \%)$ testes. Diagnostic laparoscopy was not used in any of our patients.

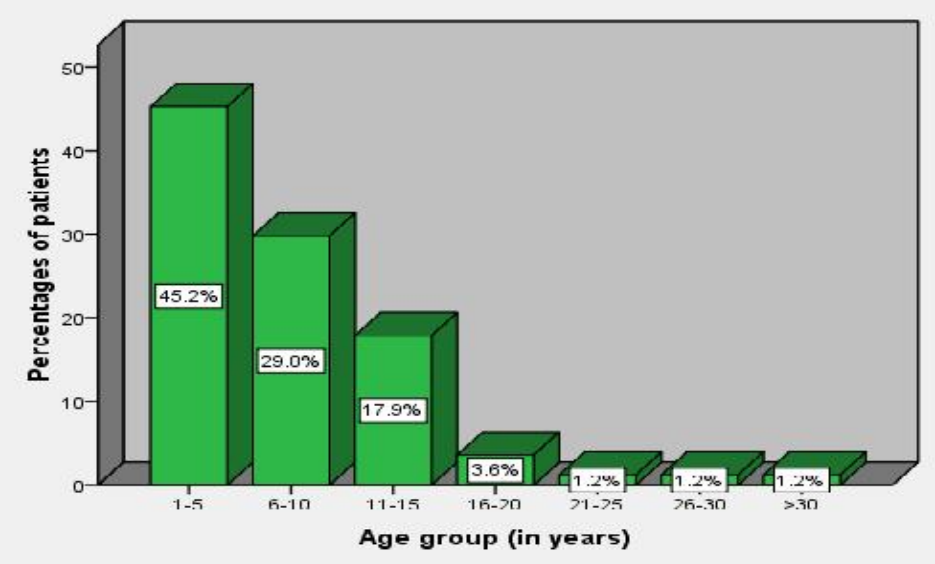

Figure 1: Distribution of patients according to age group

All patients in this study underwent surgical treatment for UDT. The median age at surgery was 8 years (+IQR of 4 to 10 years) and most of the patients, 46 (54.9\%) were aged 5 years and above at surgery. The operative techniques were through an inguinal approach for all the patients. No patient had scrotal or abdominal approaches and laparoscopic approach was not performed in any of our patients. Intraoperative assessment of testes revealed that the size of the testes was reported as normal in $62(60.8 \%)$ testes, atrophic in $16(15.7 \%)$ and small size in $9(8.8 \%)$ testes. Ten $(9.8 \%)$ testes were not found during groin exploration and in $5(4.9 \%)$ testes the size had not been recorded.

Table 1: Analysis of factors predicting the number of orchidopexy stages $(\mathrm{N}=69)$

\begin{tabular}{|c|c|c|c|}
\hline Predictor variable & Single-stage $(n=54)$ & Multistage(n= 15) & p-value \\
\hline \multicolumn{4}{|l|}{ Age at surgery (in years) } \\
\hline$\leq 5$ & $30(93.7)$ & $2(6.3)$ & \\
\hline$>5$ & $24(64.9)$ & $13(35.1)$ & 0.014 \\
\hline \multicolumn{4}{|l|}{ Clinical findings } \\
\hline Palpable & $34(89.5)$ & $4(10.5)$ & \\
\hline Non-palpable & $20(64.5)$ & $11(35 \cdot 5)$ & 0.002 \\
\hline \multicolumn{4}{|l|}{ Location } \\
\hline Superficial inguinal pouch & $27(96.4)$ & $1(3.6)$ & \\
\hline Others & $27(65.9)$ & $14(34.1)$ & 0.012 \\
\hline
\end{tabular}

The median age at surgery in patients with normal testicular size and in those with atrophic testes was 5 years (+IQR of 3 to 7 years) and 9 years (+IQR of 7 to 11 years) respectively. This age difference was found to be statistically significant $(p=0.002)$. Of the 102 testes, $54(52.9 \%)$ were found in the inguinal canal, $28(27.5 \%)$ in the superficial inguinal pouch, 10 (9.8\%) in the abdomen and $10(9.8 \%)$ 
testes were not found at surgery. Out of the 92 (90.2\%) visible testes, $69(75.0 \%)$ underwent orchidopexy (single stage in $54(78.3 \%)$ testes and multistage in $15(21.7 \%)$ testes). Orchidectomy was done in $23(25.0 \%$ ) testes (Table 1 ). Other associated surgical procedures are shown in Table 2.

Table 2: Distribution of patients according to other associated surgical procedures

\begin{tabular}{lll}
\hline Surgical procedures & Number of patients & Percentages \\
\hline Herniotomy/herniorrhaphy & 72 & 70.6 \\
Hypospadias repair & 8 & 12.9 \\
Mayo's repair & 2 & 3.2 \\
\hline
\end{tabular}

According to Multivariate logistic regression analysis, single stage procedures were more likely in patients less than 5 years, with palpable testes and those with testes located at the superficial inguinal pouch $(p<0.001)$. Late age at surgery was significantly associated with testicular atrophy $(p=0.013)$, increased complications $(p=0.002)$, number of multistage orchidectomy $(p=0.011)$ and number of orchidectomy $(p=0.001)$.

A total number of 88 testes were treated successfully giving an overall rate of $95.7 \%$. The success rate of the procedure was influenced by the original anatomical location of the UDT as shown in Table 3, thus $100 \%$ of UDT in the superficial inguinal pouch were successfully fixed in the scrotum compared to $96.3 \%$ of those in the inguinal canal and $80 \%$ of intra-abdominal testes. This was similarly reflected in the operative technique chosen, with $100 \%$ successfully fixed in the scrotum via a single stage orchidopexy compared to $86.7 \%$ successfully fixed in the scrotum via a multistage orchidopexy (Table 4). This difference was statistically significant $(p=0.011)$.

Table 3: Overall success rates by original testicular position and stages of orchidopexy

\begin{tabular}{|c|c|c|c|c|}
\hline Study variable & Response & $\begin{array}{l}\text { Total number of } \\
\text { testes }\end{array}$ & $\begin{array}{l}\text { Number of testes } \\
\text { successfully treated }\end{array}$ & $\begin{array}{l}\text { Success } \\
\text { rates (\%) }\end{array}$ \\
\hline \multirow{4}{*}{$\begin{array}{l}\text { Testicular } \\
\text { position }\end{array}$} & Inguinal canal & 54 & 52 & 96.3 \\
\hline & Superficial inguinal pouch & 28 & 28 & 100.0 \\
\hline & Intraabdominal & 10 & 8 & 80.0 \\
\hline & Total & 92 & 88 & 95.7 \\
\hline \multirow{3}{*}{$\begin{array}{l}\text { Stages } \\
\text { orchidopexy }\end{array}$} & Single stage & 54 & 54 & 100 \\
\hline & Multistage & 15 & 12 & 86.7 \\
\hline & Total & 69 & 66 & 95.7 \\
\hline
\end{tabular}

Postoperative complications were recorded in 8 patients giving a complication rate of 9.5\%. Table 4 shows distribution of patients according to postoperative complications. In this study, only 12 (14.3\%) patients were available for follow up at 12 months after discharge.

Table 4: Distribution of patients according to postoperative complications

\begin{tabular}{lll}
\hline Postoperative complications & Frequency & Percentages \\
\hline Scrotal/groin hematoma & 3 & 37.5 \\
Surgical site infection & 2 & 25.0 \\
Retracted testis necessitating repeat surgery & 1 & 12.5 \\
Chronic groin pain & 1 & 12.5 \\
Testicular atrophy & 1 & 12.5 \\
\hline
\end{tabular}




\section{Discussion}

Despite the fact that undescended testis is a congenital anomaly noticeable at birth, only $45.2 \%$ of the patients in this study presented within the first 5 years of life, with a mean age of 6 years. These findings are similar to earlier reports elsewhere (Mlayi \& Sayi., 1994; David et al., 2008), but at variance with reports from developed countries (Alsaywid, 2013). The delay in presentation is partly a reflection of the level of awareness of the people, financial constraint and none availability of trained birth attendants that can diagnose undescended testis at delivery. However, in this study we could not establish the main reasons for the delayed presentation due to the retrospective nature of the study.

Associated inguinal hernia in this study was documented in $77.4 \%$ of cases which is comparable with $75 \%$ of cases reported by Mlay \& Sayi (1994) in Tanzania. The incidence of processus vaginalis and manifest hernia in association with an undescended testis has been well described before. The patent processus vaginalis has been reported to range from $50 \%$ to $90 \%$ of patients with UDT which could harbor an occult inguinal hernia whereas $6 \%$ of clinical hernias are associated with undescended testes (Snyder \& Chaffin., 1955). The reason for this variation remains uncertain.

In the present study, UDT were discovered by the parents in $66.7 \%$ of patients and only $9.5 \%$ were discovered by birth attendants. The patients themselves and those who presented when complications such as torsion had occurred made the discovery in $21.4 \%$ cases and $2.4 \%$ respectively. This observation agrees with other studies in developing countries (Ameh \& Mbibu., 2000; David et al., 2008; Adesanya et al., 2013). In most developed countries the genitals of all male neonates are examined at birth but still UDT may be missed until the boy is examined by a school medical officer (Hutson, 1988). In developing countries such as Tanzania a school pre-entry medical examination is not routinely done hence missing a chance to detect this abnormality early. The low incidence of UDT discovered by birth attendants in this study may be explained by the fact that most of the deliveries in this region are conducted by traditional birth attendants that had no knowledge about the condition.

In the present study, some laterality in occurrence of UDT was demonstrated. The frequency of right-sided UDT was higher than left-sided UDT. Similar observation was also reported by Mlay \& Sayi (1994) in Dar es Salaam, Tanzania. The higher incidence of right-sided UDT is probably explained by later descent of right testis as opposed to the left, which is therefore more likely to result in its arrest due to mechanical factors. In Dar es Salaam, Tanzania, Mlay \& Sayi (1994) reported that more than $70 \%$ of UDT were non-palpable. The location of the impalpable testes usually presents a challenge. The importance of imaging facility such as ultrasound scan, in locating UDT cannot not be over emphasized particularly the intraabdominal and intracanalicular testes. To date, the most reliable diagnostic method for the impalpable testes is laparoscopy and definitive treatment is possible at laparoscopy in 50\% cases (Cortes et al., 1995; El-Anany et al., 2007). In our environment, only ultrasound is available and was useful in 35.3\% cases, a figure which is higher than experiences also reported by earlier workers (Mlay \& Sayi, 1994; Ameh \& Mbibu., 2000). David et al (2008) reported ultrasound to be useful in preoperatively localizing $80 \%$ of the clinically impalpable testes. No diagnostic or therapeutic laparoscopy was used in any of our patients as this facility is not available in our hospital.

In keeping with other studies done in developing countries (Mlayi \& Sayi., 1994; Ameh \& Mbibu, 2000; David et al., 2008; Adesanya et al., 2013), most of the patients in this study had surgery after the age of 5 years. This contrasts what pertains in developed countries where surgeries are routinely carried out before the age of 2 years to avert the untoward effects of the absence of the testes in the scrotum. Late age at surgery was significantly associated with increased number of 
multistage orchidectomy and orchidectomy. Due to lack of facilities for laparoscopic surgery we adopted an open approach for all the patients. Majority benefited from a single-stage orchidopexy while $21.7 \%$ required a staged treatment. Single stage orchidopexy was more likely in patients less than 5 years, with palpable testes and those with testes located at the superficial inguinal pouch as previously documented by others (David et al.,2008; Adesanya et al., 2013).

In the current study, more than fifty percent of the testes were found in the inguinal canal. This is in agreement with other studies (David et al.,2008; Adesanya et al., 2013), but at variance with Mlay \& Sayi (1994) in Tanzania and Sekabira et al (2003) in Uganda who reported superficial inguinal pouch as the most common location of the UDT. The overall success rate of $95.7 \%$ in the present study compared favorably with those reported in the literature (Abolyosr, 2006; El-Anany et al., 2007).

In this study, the follow up of our patients was generally poor as only $14.3 \%$ patients were available for follow up at 12 months after discharge. During the follow up period, one testis which was managed by retracted necessitating repeat orchidopexy. Poor follow up of patients was a major problem in this study as it was not possible to assess the long term outcome of treatment. The potential limitation of this study is the fact that information about some patients was incomplete in view of the retrospective nature of the study and this might have introduced some bias in our findings. However, despite this limitation, the study has provided local data that can help healthcare providers in the management of patients with UDT.

In conclusion, patients with UDT presented late in our hospital because diagnosis by birth attendants were rarely made even though the anomaly was obvious at birth. The parents, patients themselves and development of complications drew attention to undescended testis. The delay in presentation resulted in testicular atrophy, increase complications, number of multistage orchidopexy and orchidectomy. Health awareness campaign, thorough genital examination after birth and regular screening of toddlers for undescended testis may result in early presentation. Early diagnosis and management of the undescended testicle are needed to preserve fertility and improve early detection of testicular malignancy.

\section{Acknowledgments}

We would like to express our gratitude to all those who participated in the preparation of this manuscript. Special thanks go to the staff members of Medical records department of Bugando Medical Centre for their support during data collection

\section{References}

Abolyosr, A. (2006) Laparoscopic versus open orchiopexy in the management of abdominal testis: A descriptive study. International Journal of Urology 13, 1421-4

Adesanya, O.A., Ademuyiwa, A.O., Elebute, O.A., Ojewola, R. \& Bode, C.O. (2013) The Undescended testes in children: a prospective epidemiological study. East and Central African Journal of Surgery $18,43-49$.

Alsaywid, B.S. (2013) Surgical management of undescended testis: a two-year practice audit. Webmed Central Paediatric Surgery 4, WMC004027

Ameh, E.A. \& Mbibu, H.N. (2000) Management of undescended testes in children in Zaria, Nigeria. East African Medical Journal 77, 485-487.

Berkowitz, G., Lapinski, R., Dolgin, S., Gazella, J., Bodian, C. \& Holzman, I. (1993) Prevalence and natural history of cryptorchidism. Pediatrics 92, 44-49. 
Claude, K., Bengt, K., Ulf, H., Tina, G. \& RitzÃn, E.M. (2007) Surgical treatment of unilaterally undescended testes: testicular growth after randomization to orchiopexy at age 9 months or 3 years. Journal of Urology 178, 1589-1593.

Cortes, D., Thorup, J.M., Lenz, K., Beck, B.L. \& Nielsen, O.H. (1995) Laparoscopy in 100 consecutive patients with 128 impalpable testes. British Journal of Urology 75, 281-287.

David, L.W., David, A.B. \& John, M.P. (2004) What is the rate of spontaneous testicular descent in infants with cryptorchidism? Journal of urology 171, 849-851

David, O.O. \& lyekoretin, E. (2008) Undescended testes in a developing country: A study of the management of 71 patients. African Journal of Paediatric Surgery 5, 11-14.

Dina, C., Emma, M.K., Mette, B. \& Jorgen, T. (2008) The True Incidence of Cryptorchidism in Denmark. The Journal of Urology 179, 314-318.

Dorit, Z., Yael, I., Zehava, H., Danny, S., Ron, B. \& Itamar, A. (2006) Torsion of the cryptorchid testis" can it be salvaged? The Journal of Urology 175, 2287-2289.

El-Anany, F., Gad El-Moula, M., Abdel-Moneim, A., Abdallah, A., Takahashi, M., Kanayama, H. \& ElHaggagy, A. (2007) Laparoscopy for impalpable testis: classification-based management. Surgical Endoscopy and Other Interventional Techniques 21, 449-454

Hutson, J.M. (1988) The Surgical Examination of Children. Pd Edition, 35-64

Hutson, J.M. (1998) Undescended testis, torsion, and varicocele. In: O'Neill, J.A., Rowe, M.I., Grosfeld, J.I., Fonkalsrud, E.W., Coran, G.A. (editors). Paediatric Surgery. 5th ed. Philadelphia: Mosby Year Book Inc; 1087-109.

Hutson, J.M. \& Clarke, M.C. (2007) Current management of the undescended testicle. Seminars in Pediatric Surgery 16, 64-70.

Lindgren, B.W., Franco, I., Blick, S., Levitt, S.B., Brock, W.A., Palmer, L.S., Friedman, S.C. \& Reda, E.F. (1999) Laparoscopic Fowler-Stephens orchiopexy for the high abdominal testis. The Journal of Urology 162, 990-993.

Mlay, S.M. \& Sayi, E.N. (1994) Undescended testes in Paediatric patients at Muhimbili Medical Centre, Dar-es-Salaam. East African Medical Journal 71, 2.

Osegbe, D.N. \& Amaku, E.O. (1985) The causes of male infertility in 504 consecutive Nigerian patients. International Urology and Nephrology 17, 349-58

Sekabira, J., Kaggwa, S. \& Birabwa-Male, D. (2003) Prevalence and patterns of undescended testis among primary school pupils in Kampala, Uganda. East and Central African Journal of Surgery 8, 25-27.

Seth, A.C., Louis, J.G. \& Barry, A.K. (2006) Orchiopexy Practice Patterns in New York State From 1984 to 2002. The Journal of Urology 176, 1180-1183.

Snyder, J.W.H. \& Chaffin, L. (1955) Inguinal hernia complicated by undescended testis. American Journal of Surgery 90, 325-30

Steven, G.D. (1995) The results of surgical therapy for cryptorchidism: a literature review and analysis. The Journal of Urology 154, 1148-1152.

Taha, S.A., Abdukadar, A., Kamal, B.A. \& Anikwe, R.A. (1990) Management of an unusually high postpubertal presentation of cryptorchidism. International Surgery 75, 105-108.

Umakanth, A.K. \& Menon, P.S. (2000) Management of undescended testis. Indian Journal of Pediatrics 67, 449-454 